\title{
HYDROLOGIE
}

\section{Sur l'étude des relations entre le régime des pluies et le boisement des surfaces.}

\author{
Par Laurent RIGOTARD, Ingénieur agranome.
}

\begin{abstract}
Il existe entre le régime des pluies et le baissement des terrains des relations certaines, d'après $\mid$ mesures déjà effectuées. Ces relations sont encore bien mal connues et beaucoup d'entre elles sont seulemen soupçonnées d'exister. Il faudrait pour leur donner la valeur de lois physiques, pouvant être traduiles par di équations, un nombre élevé d'observalions rigoureuses. Jusqu'à présent les traités de météorologie se bra nent sur ce sujet d̀ des formules, des phrases vagues, Jaisant appel soit au bon sens, soit à une? observalibi déterminée souvent sommaire, ce qui est loin par conséquent de satisfaire l'esprit.

Il est donc de toute évidence nécessaire de poursuivre et de multiplier les observalions hiot faites, avant de se prononcer sur ces relations, en particulter sur les condensations qui portent - un pr malheureusement pour elles, il est vrai - le titre $d^{\prime}$ " occultes "pour nous rappeler sans cesse la profonden de notre ignorance en ce que les concerne. C'est une raison de plus d'attirer l'attenlion sur slles.
\end{abstract}

L'étude de M. Paul Descombes parue dans la Houille Blanche de novembre-décembre 1921, semble solliciter de la part des chercheurs privés, comme des groupements intéressés, une coordination d'efforts pour rechercher des lois qui régissent les condensations dites occultes, et spécialement le rôle des forêts dans la fixation du régime pluviométrique.

Tout d'abord, il est intéressant de constater qu'un premier essai de détermination d'un excédent pluvial par mètre d'altitude est présenté par l'auteur. Mais les calculs exposés nous paraissent devoir appeler certaine discussion critique. En premier lieu, nous pensons qu'il serait bon dans le calcul de ces excédents pluviaux par mètre d'altitude de ne pas utiliser de stations dont les situations géographiques et météorologiques sont aussi dissemblables que celles de Nice, Valence. D'autre part, il semble difficile de fixer l'influence des forêts dans les discordances très grandes observées : il n'y a pas, accompagnant les tableaux de coefficients calculés, une discussion des nombres obtenus dans le but de chercher et de classer les nombreuses causes des différences qui peuvent ètre indépendantes du facteur forêt.

Il est dit notamment que les stations à l'est du Rhône où la moyenne d'excédent pluvial par mètre d'altitude atteint $1 \mathrm{~m} / \mathrm{m}, 07$ au lieu de $0 \mathrm{~m} / \mathrm{m}, 83$ à l'ouest reçoivent beaucoup plus de pluie que le plateau de l'Ardèche brûlé par le soleil, faute de bois.

Il me semble difficile de discerner le rôle de la forêt dans l'état de choses constaté. Peut-être serait-il possible de déterminer un rôle certain des forêts sur de grandes plaines d'abrasion où une richesse plus grande du sol en certaines places aurait favorisé l'établissement de forêts, lesquelles alors provoquant une plus abondante chute de pluie ce qui est incontestable - auraient eu un ròle prépondérant dans la formation du modelé. La forêt aurait donc pu et pourrait fixer la position de certaines vallées. D'ailleurs il arrive cncore actuellement que la végétation forestière fixe la position de très petits cours d'eau sous nos yeux, de là nous n'hésitons pas à reconnaître son influence dans des vallées plus importantes. Mais ce parait être surtoul le rôle de fixation du sol qui est en jeu et ceci ne peut être qu'une parenthèse ; le rôle de la forêt qui est le plus difficile à soumettre au calcul, et semble.se dérober davantage à nos observations, reste ce rôle d'ordre physique, de facteur favo risant la condensation des météores aqueux. Bien que lidide: d'une influence des forêts puisse paraîtré hasardée, sị l'ok cherche à expliquer les grandes lignes du relief que la tecto. nique revendique énergiquement comme l'œuvre de forees mécaniques, cette influence peut exister dans des régions géologiquement calmes, moins bouleversées. Il est dontr regrettable que les études comparées déjà publiées, des excédents pluviaux par mètre d'altitude se rapportent an bassin du Rhône, c'est-à-dire à un bassin où le relief est dû à des mouvements importants de l'écorce terrestre De plus, la position de la vallée du Rhône étant normale la circulation générale de l'atmosphère (mouvements d'ouest en est des perturbations - voir carte des trajer. toires des dépressions, cartes des vents de France et d'Eurrope, carte des pluies de France et d'Europe), il serail utile de faire les déterminations de ces excédents pluviaur par mètre dans d'autres régịons exposées de façon aussi comparables que possible au point de vue de la circulation atmosphérique, par exemple d'axe est-ouest comme cert: taines parties des vallées de la Garonne, de la Loire. De plus, il faudrait dans ces situations météorologiquement contparables d'un même bassin choisir des surfaces dont les aceldents du relief comme aussi la composition des sols (permé. bilité, richesse chimique, etc.) soient comparables. Il serait alors possible de considérer les variations du facteur foret comme prépondérante pour expliquer les variations de la hauteur de pluie recueillie et de sa répartition dans l'alnée.

La nécessité du boisement et du reboisement n'a jamẩ fait de doute pour personne. En cherchant sans cesse det raisons nouvelles d'étendre les surfaces boisées, il importe pour ne pas jeter un trouble dans certains esprits au sujet de la grandeur comparée des facteurs en jeu dans la phy sique du globe, de ne pas expliquer certains phénomènes par la forêt seule, ni mème par la forêt simplement misê en premières lignes, avant que son rôle ait été hicn reconnt et prouvé. La science pourrait y perdre, puis par un retoll fréquent des choses, lorsque la lumière serait plus intensse c'est peut-être la forèt elle-même qui aurait à acquitter la rançon des erreurs possibles. 